\title{
Comparison between Commensurate and Non-commensurate Fractional Systems
}

\author{
Khaled HCHEICHI and Faouzi BOUANI \\ University Tunis EL Manar \\ National Engineering School of Tunis \\ Analysis, Conception and Control of Systems Laboratory, Tunis, Tunisia
}

\begin{abstract}
This article deals with fractional systems that represent better physical process and guarantee a very small number of parameters that can reduces the computation time. It focuses in particular on the state-space representation which highlights the state variables and allows to study the internal behavior of the system taking into account the initial state. Moreover, this representation adapts better to the multiple input multiple-output case. It also discusses the discretization of fractional system to finally adapt the Model Predictive Control to apply it and shows its efficiency and performance in these systems. The main objective of this article is to compare the commensurate and noncommensurate fractional models performance, calculation time and ease of use.
\end{abstract} time

Keywords-discretization; state-space; fractional; calculation

\section{INTRODUCTION}

Over the last few years, many theoretical and practical contributions showed the importance of fractional systems and their interest in different applications of modeling, identification and control of physical systems. Several control strategies have been developed and adapted for fractional systems such as the PID controller, sliding mode control and predictive control. In different disciplines such as electricity, chemistry, biology, economics, automation and signal processing. Some researchers have compared the fractional models with the classical integer models. The results have shown that fractional model represents systems with much lower number of parameters than those of integer model.

The most studied physical phenomena and most used during modeling or identification are mentioned in [1].

One of these phenomena is the attenuation of the movement of water on dikes, especially those with cavities or depressions trapping air pockets that can be compressed by water.

There is also the viscoelasticity of the materials having mechanical properties strongly dependent on the frequency over many decades of frequency, where the number of parameters is very large. As a result, calculations on the model take time and produce high order differential equations. Which is solved by using a fractional model with a number of paramtrestres very reduced in [2].

Ref [3] treated another phenomenon that requires the use of the fractional model which is the Randles model that is frequently used in the literature for modeling lead-acid batteries. This model results from a simplified solution of the electrochemical diffusion equation in the batteries (Fick's law).
To finish, the diffusion of heat in a semi-infinite environment subjected to a heat flux $q(t)$ on its surface boundary $S$. In $[4,5]$, authors demonstrate that the mathematical equations describing the unidirectional heat transfer in the environment reveal a real order of derivation of the temperature at the point of abscissa $x=0$.

Almost all articles dealing with fractional systems focus on commensurate order systems and the developed controls are based on the transfer function model. But in practice the identification programs give better results in free identification. Therefore the orders of systems can be arbitrary, this type is named non-commensurate order. This can impose problem because in the case of non-commensurate the use of the classic tools becomes imopossible, moreover the programming becomes more complicated.

This paper compares the commensurate and noncommensurate fractional system discussing the advantages and the disadvantages of each model. The models used in this paper are in the form of a state-sapce representations that make it easier to study the internal behavior of systems and adapt better to MIMO systems.

The first section of this article quotes the different representations of fractional systems as well as the transition between them. A second section explores the method used to pass from a continuous model to a discrete model. Section 3 will present the predictive control that will be applied on the fractional model. In Section 5 we have implemented different methods to compare commensurate and non-commensurate fractional systems.

\section{General Fractional System Representation}

A generalized fractional system can be represented by the following equation $[6,7]$ :

$$
y(t)+\sum_{i=1}^{n} a_{i} D^{\alpha_{i}} y(t)=\sum_{i=1}^{m} b_{i} D^{\beta_{i}} u(t)
$$

As the system is relaxed $y(t)=u(t)=0$ for $t \leqslant 0$, the Laplace transform of $D^{\alpha_{i}} y(t)$ and $D^{\beta_{i}} u(t)$ are respectively $s^{\alpha_{i}} Y(s)$ and $s^{\beta_{i}} U(s)$, where $Y(s)$ and $U(s)$ are the Laplace transforms of $y(t)$ and $u(t)$. Applying the Laplace transformation to (1) we obtain:

$$
Y(s)+\sum_{i=1}^{n} a_{i} s^{\alpha_{i}} Y(s)=\sum_{i=1}^{m} b_{i} s^{\beta_{i}} U(s)
$$


The transfer function can be deduced from the previous equation:

$$
G(s)=\frac{Y(s)}{U(s)}=\frac{\sum_{i=1}^{m} b_{i} s^{\beta_{i}}}{1+\sum_{i=1}^{n} a_{i} s^{\alpha_{i}}}
$$

The generalized state space model corresponding to the multivariable transfer function is:

$$
\begin{cases}D^{(\alpha)}(x) & =A x+B u \\ y & =C x+D u\end{cases}
$$

Where:

$$
D^{(\alpha)}(x)=\left[\frac{d^{\alpha_{1}}}{d t^{\alpha_{1}}} x_{1}, \frac{d^{\alpha_{2}}}{d t^{\alpha_{2}}} x_{2} \cdots \frac{d^{\alpha_{n}}}{d t^{\alpha_{n}}} x_{n}\right]^{T}
$$

The passage from the state-space representation to the transfer-function representation can be deduced as in the integer case by taking the Laplace transform and considering the zero initial conditions [8], it is given by:

$$
G(s)=C\left[\left(s^{(\alpha)} I_{n}-A\right)^{-1}\right] B+D
$$

On the other hand, to go from the transfer function to the state representation there is a difference. This difference is due to the presence of fractional derivation orders that must be taken into account during transformation. As mentioned, the transfer function of fractional systems is represented as follows:

$$
G(s)=\frac{b_{m} s^{\beta_{m}}+b_{m-1} s^{\beta_{m-1}}+\cdots+b_{1} s^{\beta_{1}}+b_{0}}{s^{\alpha_{n}}+a_{1} s^{\alpha_{n-1}}+\cdots+a_{n-1} s^{\alpha_{1}}+a_{n}}
$$

With

$$
\alpha_{n}>\alpha_{n-1}>\cdots>\alpha_{2}>\alpha_{1}
$$

and

$$
\beta_{m}>\beta_{m-1}>\cdots>\beta_{2}>\beta_{1}
$$

Let $\tilde{\alpha}$ be the vector obtained from the concatenation of the fractional numbers $\alpha_{i}$ and $\beta_{i}$ such that:

$$
\tilde{\alpha}=\left[\begin{array}{llllll}
\tilde{\alpha}_{n+m} & \tilde{\alpha}_{n+m-1} & \tilde{\alpha}_{n+m-2} & \cdots & \tilde{\alpha}_{2} & \tilde{\alpha}_{1}
\end{array}\right]
$$

With

$$
\tilde{\alpha}_{n+m}>\tilde{\alpha}_{n+m-1}>\tilde{\alpha}_{n+m-2}>\cdots>\tilde{\alpha}_{2}>\tilde{\alpha}_{1}
$$

Let consider the continuous state-space model given by:

$$
\begin{cases}D^{\gamma}(x) & =A_{c} x+B_{c} u \\ y & =C_{c} x\end{cases}
$$

with

$$
A_{c}=\left(\begin{array}{ccccc}
0 & 1 & \cdots & 0 & 0 \\
0 & 0 & & 0 & 0 \\
\vdots & & \ddots & & \vdots \\
0 & 0 & & 0 & 1 \\
\tilde{a}_{n+m} & \tilde{a}_{n+m-1} & \cdots & \tilde{a}_{2} & \tilde{a}_{1}
\end{array}\right), \quad B_{c}=\left(\begin{array}{c}
0 \\
0 \\
\vdots \\
0 \\
1
\end{array}\right)
$$

$$
C_{c}=\left(\begin{array}{lllll}
\tilde{c}_{1} & \tilde{c}_{2} & \cdots & \tilde{c}_{n+m-1} & \tilde{c}_{n+m}
\end{array}\right)
$$

and

$$
D^{\gamma} x=\left[\begin{array}{lll}
D^{\tilde{\alpha_{1}}} x_{1} & \cdots & D^{\left(\tilde{\alpha}_{n+m}-\tilde{\alpha}_{n+m-1}\right)} x_{n+m}
\end{array}\right]
$$

Where the corresponding transfer function model $H(s)$ is given by:

$$
\begin{aligned}
H(s)= & \frac{\tilde{c}_{1}+\tilde{c}_{2} s^{\tilde{\alpha}_{1}}+\tilde{c}_{3} s^{\tilde{\alpha}_{1}+\tilde{\alpha}_{2}}+\cdots}{s^{\tilde{\alpha}_{1}+\tilde{\alpha}_{2}+\cdots+\tilde{\alpha}_{n+m}}+\cdots} \\
& \frac{\cdots+\tilde{c}_{n+m} s^{\tilde{\alpha}_{1}+\tilde{\alpha}_{2}+\cdots+\tilde{\alpha}_{n+m-1}}}{\cdots+\tilde{a}_{n+m-2} s^{\tilde{\alpha}_{1}+\tilde{\alpha}_{2}}+\tilde{a}_{n+m-1} s^{\tilde{\alpha}_{1}} \tilde{a}_{n+m}}
\end{aligned}
$$

Since $H(s)$ numerator and denominator contain $(n+m)$ terms, it is then sufficient to sort them in order to isolate $m$ terms for witch the fractional orders correspond to those of $G(s)$ numerator and $n$ terms for witch the fractional orders correspond to those of $G(s)$ denominator. The procedure of selection of the terms $\tilde{c}_{i}$ and $\tilde{a}_{i}$ is summarized in (12).

$$
\begin{aligned}
& \left\{\begin{array}{l}
\tilde{a}_{n+m}=a_{n} \quad \tilde{c}_{1}=b_{0} \\
\text { if } \tilde{\alpha}_{i}=\beta_{j} \text { then } \tilde{c}_{i+1}=b_{j} \text { and } \tilde{a}_{n+m-i}=0 \\
\text { if } \tilde{\alpha}_{i}=\alpha_{j} \text { then } \tilde{c}_{i+1}=0 \text { and } \tilde{a}_{n+m-i}=a_{n-j}
\end{array}\right. \\
& i=1, \cdots, n+m-1 \text { and } j=1, \cdots, n+m-1
\end{aligned}
$$

In this case of generalized fractional systems, the number of state variables is equal to the sum of dimensions of the numerator and denominator polynomials of the transfer function $(n+m)$.

\section{Discritization of Fractional State-Space MODEL}

Contrary to the commensurate case, discretization in the case of non-commensurate fractional systems must take into account the plurality of derivations of state variables.

To move from a continuous model to a discrete model it is necessary to use this approximation $[9,10,11]$ :

$$
D^{\gamma} x(t)=\frac{1}{T_{s}^{\gamma}} \sum_{j=0}^{p}(-1)^{j}\left(\begin{array}{l}
\gamma \\
j
\end{array}\right) x\left((k-j) T_{s}\right)
$$

Let's assume that the vector of continuous model derivation $\gamma=\left[\begin{array}{llll}\gamma_{1} & \gamma_{2} & \cdots & \gamma_{r}\end{array}\right]^{T}, T_{s}$ is the sampling time and $p \in \mathbb{N}$ is the number of past samples with which the derivation was computed.

If $(i=1, \cdots, r)$, the term $\left(\begin{array}{l}\gamma \\ j\end{array}\right)$ can be written as follows:

$$
\begin{aligned}
& \left(\begin{array}{l}
\gamma \\
j
\end{array}\right)^{T}=\left[\begin{array}{llll}
\left(\begin{array}{c}
\gamma_{1} \\
j
\end{array}\right) & \left(\begin{array}{c}
\gamma_{2} \\
j
\end{array}\right) & \cdots & \left(\begin{array}{c}
\gamma_{r} \\
j
\end{array}\right)
\end{array}\right] \\
& \left(\begin{array}{c}
\gamma_{i} \\
j
\end{array}\right)=\left\{\begin{array}{lll}
1 & \text { for } & j=0 \\
\frac{\gamma_{i}\left(\gamma_{i}-1\right) \ldots\left(\gamma_{i}-j+1\right)}{j !} & \text { for } & j>0
\end{array}\right.
\end{aligned}
$$


By multiplying (13) by $T_{s}^{\gamma}$ and developing the terms of $j=0$ and $j=1$ the following result is found:

$$
\begin{aligned}
T_{s}^{\gamma} D^{\gamma} x(t)= & x\left(k T_{s}\right)-\gamma x\left((k-1) T_{s}\right)+ \\
& +\sum_{j=2}^{p}(-1)^{j}\left(\begin{array}{l}
\gamma \\
j
\end{array}\right) x\left((k-j) T_{s}\right)
\end{aligned}
$$

Now let consider the following continuous fractional statespace model [12]:

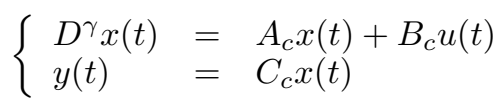

With $A_{c} \in \mathbb{R}^{r \times r}, B_{c} \in \mathbb{R}^{r \times 1}$ and $C_{c} \in \mathbb{R}^{1 \times r}$ are the state matrices of the continuous fractional model and $r$ is the number of varibales in state-space model.

$$
\begin{aligned}
& T_{s}^{\gamma} A_{c} x\left(k T_{s}\right)-x\left(k T_{s}\right)=-\gamma x\left((k-1) T_{s}\right)+ \\
& \quad+\sum_{j=2}^{p}(-1)^{j}\left(\begin{array}{l}
\gamma \\
j
\end{array}\right) x\left((k-j) T_{s}\right)-B_{c} T_{s} u\left(k T_{s}\right)
\end{aligned}
$$

Note that $I_{r} \in \mathbb{R}^{r \times r}$ the identity matrix and $T_{s}^{\gamma}$ the diagonal matrix filled by $\left(T_{s}^{\gamma_{1}} \cdots T_{s}^{\gamma_{r}}\right)$.

To facilitate writing, note

$$
\mathbb{Z}=\left(T_{s}^{\gamma} A_{c}-I_{r}\right)^{-1}
$$

$$
\begin{aligned}
x\left(k T_{s}\right)= & -\mathbb{Z} \gamma x\left((k-1) T_{s}\right)+\mathbb{Z} \sum_{j=2}^{p}(-1)^{j}\left(\begin{array}{l}
\gamma \\
j
\end{array}\right) x\left((k-j) T_{s}\right) \\
& -\mathbb{Z} B_{c} T_{s}^{\gamma} u\left(k T_{s}\right)
\end{aligned}
$$

and with $(i=1, \cdots, r)$

$$
c_{j}=\operatorname{diag}\left\{(-1)^{j}\left(\begin{array}{c}
\gamma_{i} \\
j
\end{array}\right)\right.
$$

The above equation can be written as:

$$
x(k)=\mathbb{Z} c_{1} x(k-1)+\mathbb{Z} \sum_{j=2}^{p} c_{j} x(k-j)-\mathbb{Z} B_{c} T_{s}^{\gamma} u(k)
$$

To simplify the equation:

$$
A_{j}=\mathbb{Z} c_{j}
$$

By expanding all terms and simplifying, (22) becomes in the form:

$x(k)=A_{1} x(k-1)+A_{2} x(k-2)+\cdots+A_{k} x(0)-\mathbb{Z} B_{c} T_{s}^{\gamma} u(k)$

The system can therefore be described by a discrete statespace representation [13]:

$$
\left\{\begin{array}{l}
X_{d}(k+1)=A_{d} X_{d}(k)+B_{d} u(k) \\
y(k)=C_{d} X_{d}(k)
\end{array}\right.
$$

With

$$
\begin{aligned}
& A_{d}=\left(\begin{array}{cccc}
A_{1} & A_{2} & \cdots & A_{p-1} \\
I & 0 & \cdots & 0 \\
0 & I & \cdots & 0 \\
\vdots & \vdots & \ddots & \vdots \\
0 & \cdots & I & 0
\end{array}\right), \quad B_{d}=\left(\begin{array}{c}
-\mathbb{Z} B_{c} T_{s}^{\gamma} \\
0 \\
\vdots \\
0
\end{array}\right), \\
& X_{d}(k+1)=\left(\begin{array}{c}
x(k+1) \\
x(k) \\
\vdots \\
x(k-p+1)
\end{array}\right) \quad \text { and } \\
& X_{d}(k)=\left(\begin{array}{c}
x(k) \\
x(k-1) \\
\vdots \\
x(k-p)
\end{array}\right)
\end{aligned}
$$

with $u, y, X_{d}$ are respectively the input, output and variables state of the process. $p$ is the number of past iterations which the system takes into account for calculating a variable, $A_{d} \in \mathbb{R}^{r p \times r p}, B_{d} \in \mathbb{R}^{r p \times 1}, C_{d} \in \mathbb{R}^{1 \times r p}$ and $X_{d} \in \mathbb{R}^{r p \times 1}$.

By increasing the number of iterations taken into account $p$ the computation time increases, so it is reasonable to choose a number large enough to represent the system correctly but not too large to reduce the calculation time.

\section{Fractional Model Predictive Control}

The principle of the predictive control is to create an anticipatory effect for the system with respecting the trajectory to follow known in advance, based on the prediction of the future behavior of the system and minimizing the gap of these predictions to the trajectory and by minimizing a certain cost function $J$, while respecting operating constraints $[14,15,16]$.

This section will develop a predictive control from the discrete fractional state-space model described in previous section. For that we will make a variable change : $\Delta X_{d}(k)=$ $X_{d}(k)-X_{d}(k-1)$ the input variable difference: $\Delta u(k)=$ $u(k)-u(k-1)$, and using it in (25) this transformation is found:

$$
\Delta X_{d}(k+1)=A_{d} \Delta X_{d}(k)+B_{d} \Delta u(k)
$$

The new state variable vector is:

$$
X(k)=\left[\Delta X_{d}(k)^{T} y(k)\right]^{T}
$$

with $y(k)$ is the output and:

$$
y(k+1)-y(k)=C_{d} A_{d} \Delta X_{d}(k)+C_{d} B_{d} \Delta u(k)
$$

The system can be written in the form:

$$
\begin{aligned}
& \left\{\begin{array}{l}
X(k+1)=A X(k)+B \Delta u(k) \\
y(k)=C X(k)
\end{array}\right. \\
& A=\left(\begin{array}{cc}
A_{d} & 0_{d}^{T} \\
C_{d} A_{d} & 1
\end{array}\right) ; B=\left(\begin{array}{c}
B_{d} \\
C_{d} B_{d}
\end{array}\right) ;
\end{aligned}
$$




$$
C=\left(0_{d} \quad 1\right) ; 0_{d} \in \mathbb{R}^{1 \times r p}
$$

Future state variables can be predicted and written in the form:

$$
\left\{\begin{aligned}
X(k+1)= & A X(k)+B \Delta u(k) \\
X(k+2)= & A X(k+1)+B \Delta u(k+1) \\
= & A^{2} X(k)+A B \Delta u(k)+B \Delta u(k+1) \\
\vdots & \\
X\left(k+H_{p}\right)= & A^{H_{p}} X(k)+A^{H_{p}-1} B \Delta u(k)+ \\
& A^{H_{p}-2} B \Delta u(k+1)+\cdots \\
& +A^{H_{p}-H_{c}} B \Delta u\left(k+H_{c}-1\right)
\end{aligned}\right.
$$

Based on (29) future system outputs can be predicted:

$$
\left\{\begin{aligned}
y(k+1)= & C A X(k)+C B \Delta u(k) \\
y(k+2)= & C A X(k+1)+C B \Delta u(k+1) \\
= & C A^{2} X(k)+C A B \Delta u(k)+ \\
& C B \Delta u(k+1) \\
\vdots & \\
y\left(k+H_{p}\right)= & C A^{H_{p}} X(k)+C A^{H_{p}-1} B \Delta u(k)+ \\
& C A^{H_{p}-2} B \Delta u(k+1)+\cdots \\
& +C A^{H_{p}-H_{c}} B \Delta u\left(k+H_{c}-1\right)
\end{aligned}\right.
$$

$H_{p}$ and $H_{c}$ are respectively the prediction horizon and the control horizon with $H_{p} \geq H_{c}$. Assume the vector $Y$ which contains $H_{p}$ system's predicted future outputs and $\Delta u$ contains $H_{c}$ future controls:

$$
\begin{gathered}
Y^{T}=\left[y(k+1) y(k+2) \cdots y\left(k+H_{p}\right)\right] \\
\Delta u^{T}=\left[\Delta u(k) \Delta u(k+1) \cdots \Delta u\left(k+H_{c}-1\right)\right]
\end{gathered}
$$

The vector $Y$ can also be written as :

$$
\begin{gathered}
Y=F X(k)+\Phi \Delta u \\
F=\left(\begin{array}{c}
C A \\
C A^{2} \\
C A^{3} \\
\vdots \\
C A^{H_{p}}
\end{array}\right) \\
\Phi^{T}=\left(\begin{array}{ccccc}
C B & C A B & C A^{2} B & \cdots & C A^{H_{p}-1} B \\
0 & C B & C A B & \cdots & C A^{H_{p}-2} B \\
0 & 0 & C B & \cdots & C A^{H_{p}-3} B \\
\vdots & \vdots & \vdots & \ddots & \vdots \\
0 & 0 & 0 & & C A^{H_{p}-H_{c}} B
\end{array}\right)
\end{gathered}
$$

The aim of predictive control is to find the control vector $\Delta u$ which forces the system's output $y$ to follow the setpoint $y_{s}$. In order to achieve this we must optimize a criterion $J$ which represents the control objective:

$$
J=\sum_{i=1}^{H_{p}}\left(y_{s}(k+i)-y(k+i)\right)^{2}+\lambda \sum_{i=0}^{H_{c}-1} \Delta u^{2}(k+i)
$$

The criterion $J$ can be written in matrix form:

$$
J=\left(Y_{s}-Y\right)^{T}\left(Y_{s}-Y\right)+\Delta u^{T} \lambda \Delta u
$$

With $Y_{s}^{T}=\left[y_{s}(k+1) y_{s}(k+2) \cdots y_{s}\left(k+H_{p}\right)\right]$ is the vector filled by the future values of the set-points and $\lambda$ is weight coefficient on the control.

By minimizing $J$ we obtain optimal control sequence [17]:

$$
\Delta u=\left(\Phi^{T} \Phi+\lambda I\right)^{-1} \Phi^{T}\left(Y_{s}-F X(k)\right)
$$

\section{Simulation Results}

In this section the matlab FOMCON toolbox is used to find a continuous fractional transfer function from the inputoutput data of a thermal system. The input signal used for identification is shown in the Fig.1. In this case we chose a free identification to find the better result.

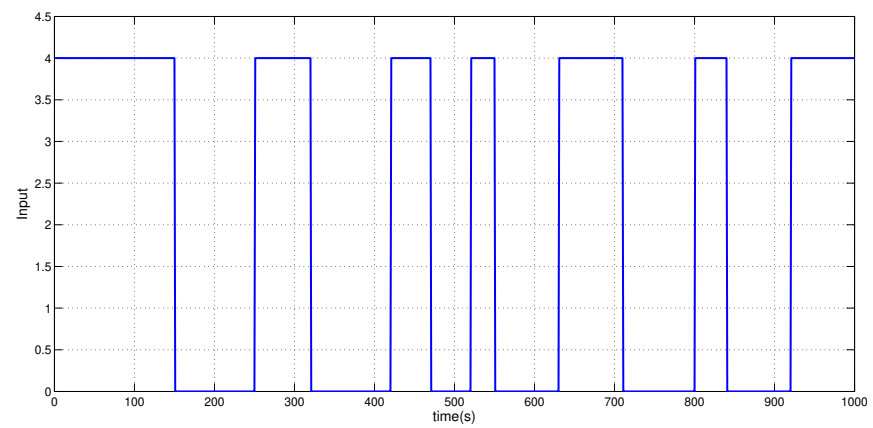

Fig. 1. System input.

The result obtained is compared with the output signal used in the identification, the Fig.2 illustrates the two signals as well as the error.

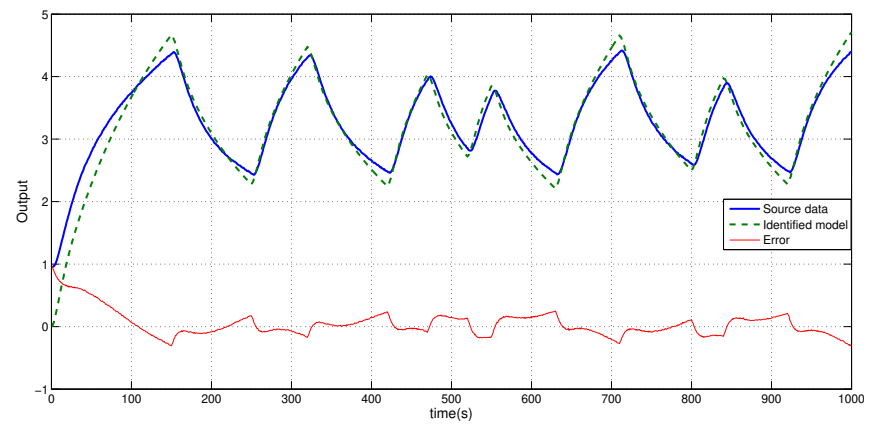

Fig. 2. Identification result.

This method gaves as result a continuous fractional transfer function (7)

$$
G(s)=\frac{b_{0}}{s^{2.46}+\tilde{a}_{1} s^{1.97}+\tilde{a}_{2} s^{1.39}+\tilde{a}_{3} s^{0.97}+\tilde{a}_{4} s^{0.88}+\tilde{a}_{5}}
$$


So the parameters are deduced: $b_{0}=-0.0041, \tilde{a}_{1}=$ $-1.5977, \tilde{a}_{2}=0.551, \tilde{a}_{3}=-0.3517, \tilde{a}_{4}=-0.0011$ and $\tilde{a}_{5}=-0.002$.

By applying the above method, the founded result is the fractional state-space representation for previous transfer function and which is described by the matrices:

$$
\begin{gathered}
A_{c}=\left(\begin{array}{ccccc}
0 & 1 & 0 & 0 & 0 \\
0 & 0 & 1 & 0 & 0 \\
0 & 0 & 0 & 1 & 0 \\
0 & 0 & 0 & 0 & 1 \\
0.002 & 0.0011 & 0.3517 & -0.551 & 1.5977
\end{array}\right), \\
B_{c}=\left(\begin{array}{l}
0 \\
0 \\
0 \\
0 \\
1
\end{array}\right), \quad C_{c}=\left(\begin{array}{lllll}
-0.0041 & 0 & 0 & 0 & 0
\end{array}\right) \\
\tilde{\alpha}_{1}=0.88, \tilde{\alpha}_{2}=0.97, \tilde{\alpha}_{3}=1.39, \tilde{\alpha}_{4}=1.97 \text { and } \tilde{\alpha}_{5}=
\end{gathered}
$$
2.46 .

and using (8) and (10) we can fill the vector $\gamma$ by :

$$
\gamma=\left(\begin{array}{c}
\tilde{\alpha}_{1} \\
\tilde{\alpha}_{2}-\tilde{\alpha}_{1} \\
\tilde{\alpha}_{3}-\tilde{\alpha}_{2} \\
\tilde{\alpha}_{4}-\tilde{\alpha}_{3} \\
\tilde{\alpha}_{5}-\tilde{\alpha}_{4}
\end{array}\right), \quad \text { then } \quad \gamma=\left(\begin{array}{c}
0.88 \\
0.09 \\
0.42 \\
0.58 \\
0.49
\end{array}\right)
$$

From transfer function (37) the commensurate model that can be found by matlab FOMCON toolbox has a very high order, in this case $r_{c}=246$ and $\alpha=0.01$ With $A_{c_{c}} \in \mathbb{R}^{r_{c} \times r_{c}}$, $B_{c_{c}} \in \mathbb{R}^{r_{c} \times 1}$ and $C_{c_{c}} \in \mathbb{R}^{1 \times r_{c}}$ are the state matrices of the continuous fractional model, $\alpha$ is the commensurate order and $r_{c}$ is the number of varibales in state-space commensurate model.

For discretization the chosen sampling period is $T_{s}=5 \mathrm{~s}$ and the history will be limited to 20 past values so $\mathrm{p}=20$, these parameters will be the same for the rest of this paper.

In what follows (Fractional Model Predictive Control) FMPC will be applied for both models, the necessary parameters for the FMPC will be fixed for this section $H_{p}=10$, $H_{c}=1$ and $\lambda=2$.

The system output (temperature) and the set-point are shown in the Fig. 3 while the Fig.4 shows the control signal generated by the FMPC. A disturbance is added to the system in the interval of time $k \in[120,150]$ to test the ability of FMPC to anticipate it, the amplitude of this disturbance is $+10 \%$.

Fig. 3 compares the output of the non-comensurate model with the commensurate model, these two models are deduced from the same transfer function presented by (37).

The Fig. 3 and Fig. 4 show that the FMPC is able to mitigate the effect of the disturbance on the output. On the other hand the Fig.5 shows peaks with a very large amplitude which appears in the control increment signal.

The figures show that the predictive control is able to force the system to follow the set-point in both cases, but it is clear

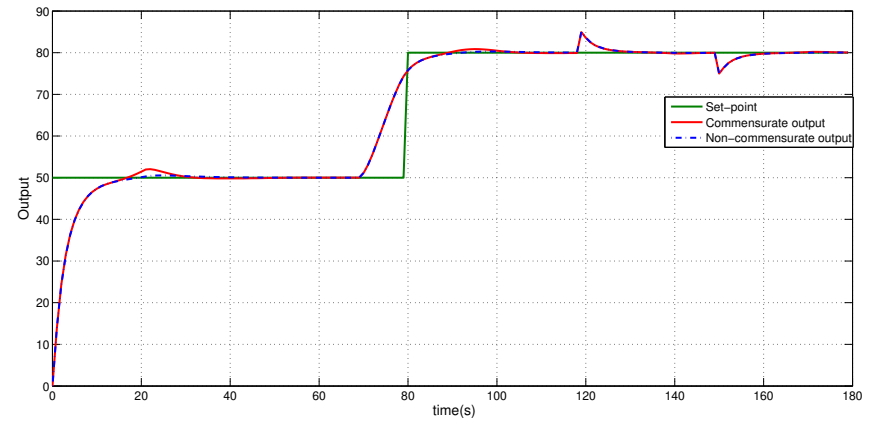

Fig. 3. Output with disturbance.

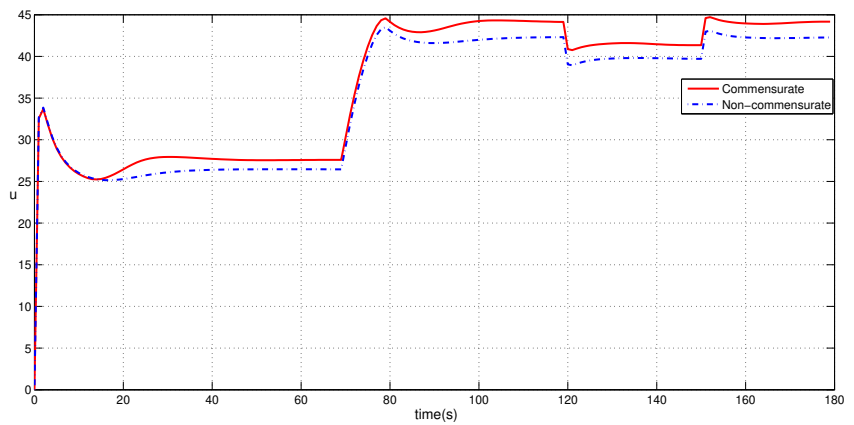

Fig. 4. Control with disturbance.

that for the non-commensurate model the control expends less energy to achieve it.

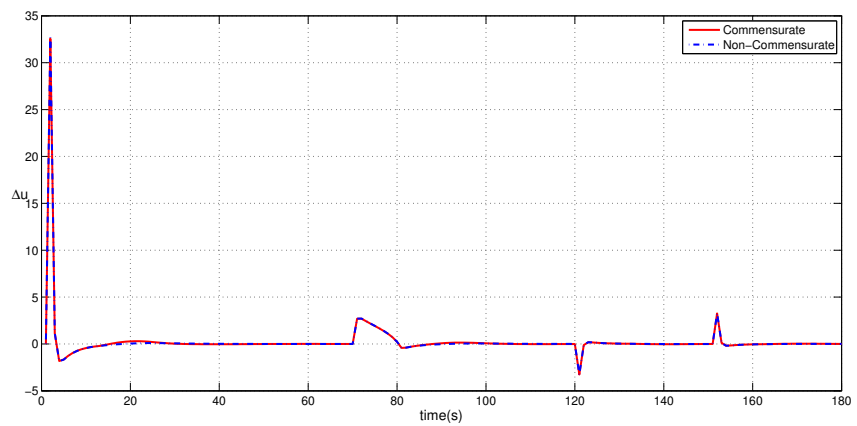

Fig. 5. Control increment with disturbance.

In the Fig.5, the control increases by a large amount instantly. To cancel this undesirable spike we will add a constraint to the calculation of control.

The principle of constraint control $[18,19]$ is to add to the main problem one or more conditions, it is therefore necessary to minimize criterion $J$ while respecting these conditions. The most used constraint form is the one on the rate of change of the control variables $\Delta u(k)$. Suppose that for a single-input system the upper limit is $\Delta u_{\max }$ and the lower limit is $\Delta u_{\min }$. The constraints are specified in the form:

$$
\Delta u_{\min } \leq \Delta u(k) \leq \Delta u_{\max }
$$

Assuming that the control variable $\Delta u(k)$ can only increase or decrease in a unit of magnitude less than 2.5, the operational constraint is:

$$
-2.5 \leq \Delta u(k) \leq 2.5
$$


Now let's test the performance of the system under constraint and with a disturbance of amplitude $+10 \%$ in the interval of time $k \in[120,150]$, the constraint is the same used before.

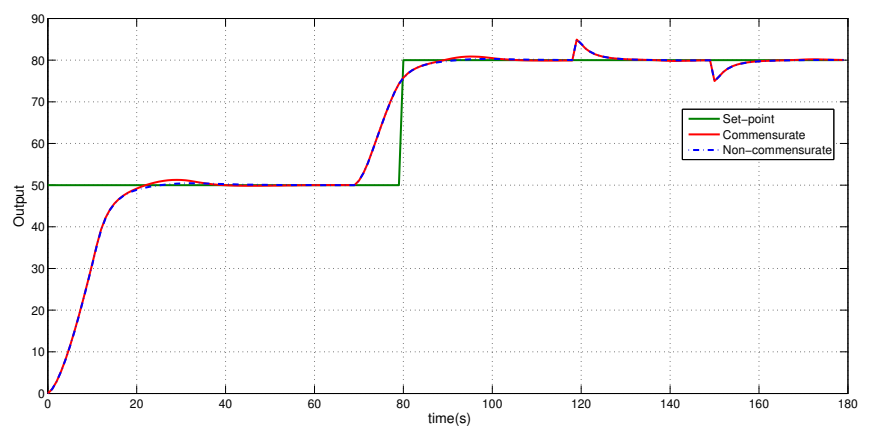

Fig. 6. Output with constraint and disturbance.

Even under constraint the FMPC can ensure that the output (temperature) follows the set-point. Constrains on the control increment guarantees that there is no peaks in the control signal. In return, the output porsuite becomes slower as shown in the Fig.6.

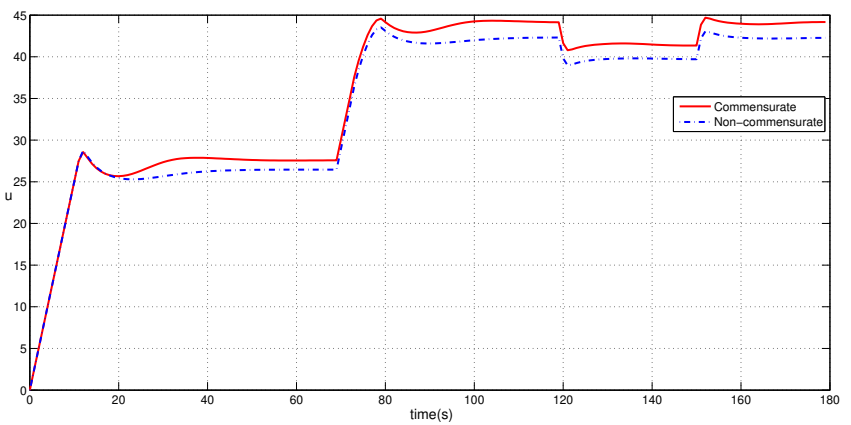

Fig. 7. Control with constraint and disturbance.

The choice of the the interval $\left[\Delta u_{\min }, \Delta u_{\max }\right]$ is very important because if the interval is too wide the condition will not be taken into account when minimizing criterion $J$, and if the interval is too small the control will no longer be able to bring the output to follow the set-point, even if it happen the system will be too slow.

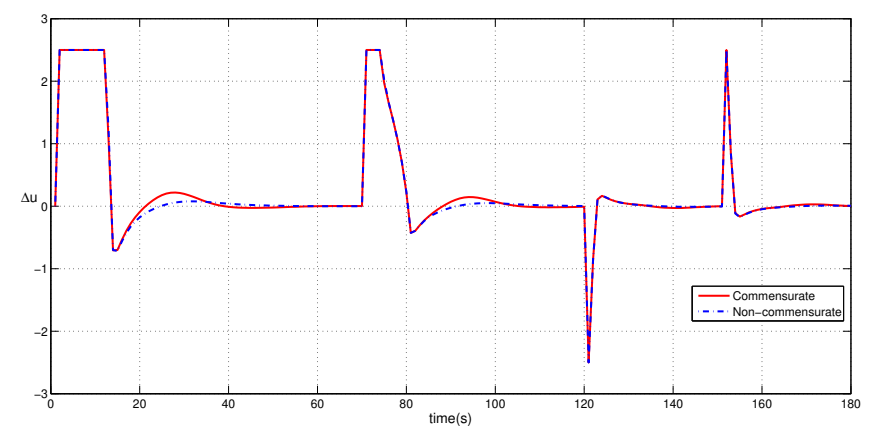

Fig. 8. Control increment with constraint and disturbance.

Fig.7 and Fig.8 show that the constraint is taken into account in optimisation and show that the non-commensurate model still expends less energy than the commensurate model.

One of the disadvantages of fractional systems is the increase of the dimensions of the matrices at each iteration. For example if $p=20$, the calculation of the state variables will depend on 20 previous values. The dimensions of the matrices will increase to multiply by $p$. This increase has a negative effect on the calculation time which increases considerably.

Now let's focus on the calculation time for both models. This part compares the time spent on each iteration to calculate the control increment $\Delta u$ for different value of $p$. In this simulation the used processor is a $i 3$ with $1.9 \mathrm{GHz}$ frequency, the results are presented in Table I:

TABle I. CAlculate Time (MS)

\begin{tabular}{|r|c|c|c|c|}
\hline $\mathrm{p}$ & 20 & 50 & 100 & 180 \\
\hline Non-commensurate & 22.7 & 23.6 & 27.9 & 30.5 \\
\hline Commensurate & 53.2 & 56.1 & 61.5 & 74.1 \\
\hline
\end{tabular}

Even if fractional models better represent physical systems, their use can be complicated. The commensurate model has a very high order $r_{c}=246$ whereas in the case noncommensurate model $r=5$, this difference is manifested in the computation time.

These results show that the use of the commensurate model can provide the same performance as non-commensurate model with a longer computing time. The non-commensurate model consecutively reduces the calculation time for the control because the matrices have a smaller dimension. On the other hand, the determination of a non-commensurate model is more complicated than the commensurate model.

\section{CONCLUSION}

The use of fractional models becomes more and more frequent given the efficiency they provide in the discription of certain physical systems. Nevertheless it remains a little difficult to handle. The majority of research $[20,21,22]$ deals with commensurate fractional models that have proved efficiency at describing several physical phenomena, but the major inconvenience is that computation time increases in discrete models because of the use of history in the calculation. This article has shown that the limitation of the used history and the use of non-commensurate models in modeling or identification can remedy this problem. It has also adapted predictive control to apply it to a non-commensurate fractional system. The importance of this work is that it deals with the state-space representation of the non-commensurate fractional systems from identification to control. It also compares the use of commensurate and non-commensurate fractional systems and explores the advantages and disadvantages of each model. At first it introduced the transition between the transfer-function and the state-space representation in the non-commensurate fractional case. Then for the same kind of system, it explained the discretization and we closed by a comparison with the commensurate model in therm of performance and computation time.

\section{REFERENCES}

[1] A. Oustaloup, J. Sabatier, P. Lanusse, et al. "An overview of the CRONE approach in system analysis, modeling and identification, observation and control" IFAC Proceedings Volumes: 17th IFAC World Congress, 2008, 41 (2): 14254 - 14265 
[2] H. Sun, Y. Zhang, D. Baleanu, W. Chen and Y. Chen "A new collection of real world applications of fractional calculus in science and engineering" Communications in Nonlinear Science and Numerical Simulation, 2018, 64: 213 - 231

[3] T.J. Machado, M. Silva, R. Barbosa, et al. "Some Applications of Fractional Calculus in Engineering" Mathematical Problems in Engineering 2010: 34 pages

[4] S. Blasiak "Time-fractional heat transfer equations in modeling of the non-contacting face seals" International Journal of Heat and Mass Transfer, 2016, 100: $79-88$

[5] L. Ouhsaine, Y. Boukal, M. El-Ganaoui et al. "A General fractionalorder heat transfer model for Photovoltaic/Thermal hybrid systems and its observer design" Energy Procedia, 2017, 139: 49 - 54

[6] C.F. Lorenzo and T.T. Hartley "Initialization in fractional order systems" In: European Control Conference (ECC), Porto, Portugal, September 2001, pp. 1471-1476

[7] A. Rhouma and F. Bouani "Robust model predictive control of uncertain fractional systems: a thermal application" IET Control Theory Applications, 2014, 8 (17): 1986-1994

[8] C.A. Monje, Y. Chen, B.M. Vinagre, D. Xue and V.Feliu-Batlle, (2010) "Fractional-order Systems and Controls: Fundamentals and Applications." London: Springer-Verlag, 1st edn

[9] Z. Belkhatir and T.M. Laleg-Kirati "Parameters and fractional differentiation orders estimation for linear continuous-time non-commensurate fractional order systems" Systems and Control Letters , 2018, 115: 26 $-33$

[10] A. Dzieliǹski and D. Sierociuk "Adaptive Feedback Control of Fractional Order Discrete State-Space Systems." In: International Conference on Computational Intelligence for Modelling, Control and Automation and International Conference on Intelligent Agents, Web Technologies and Internet Commerce (CIMCA-IAWTIC'06, Vienna, Austria, December 2011, pp. 1-6

[11] P. Ostalczyki and D. Mozyrska "The second form of the variable-, fractional-order discrete-time integrator." In: 21st International Conference on Methods and Models in Automation and Robotics (MMAR), Miedzyzdroje, Poland, August 2016, pp. 859-864

[12] A. Dzieliǹski and D. Sierociuk "Ultracapacitor modelling and con- trol using discrete fractional order state-space models and Fractional Kalman Filters." In: European Control Conference (ECC), Kos, Greece, July 2007, pp. 2916-2922

[13] M. Abedini, M.A. Nojoumian, H.Salarieh and A. Meghdari "Model reference adaptive control in fractional order systems using discretetime approximation methods" Communications in Nonlinear Science and Numerical Simulation, 2015, 25 (1-3): 27 - 40

[14] H. Aboukheir "Predictive Control of Fractional Order Systems" IFAC Proceedings Volumes, 2012, 45 (13): 622-626

[15] M.M. Joshi, V.A. Vyawahare and M.D. Patil "Model predictive control for fractional-order system a modeling and approximation based analysis." In: International Conference on Simulation and Modeling Methodologies, Technologies and Applications (SIMULTECH), Vienna, Austria, August 2014, pp. 361-372

[16] J.B. Rawlings "Tutorial overview of model predictive control" IEEE Control Systems, 2000, 20 (3): 38-52

[17] A. Rhouma, F. Bouani, B. Bouzouita and M. Ksouri "Model Predictive Control of Fractional Order Systems" Journal of Computational and Nonlinear Dynamics, 2014, 9: 310111-310117

[18] B. Sikora and J. Klamka "Constrained controllability of fractional linear systems with delays in control" Systems and Control Letters, 2017, 106: $9-15$

[19] Q. Zou, Q. Jin and R. Zhang "Design of fractional order predictive functional control for fractional industrial processes" Chemometrics and Intelligent Laboratory Systems, 2016, 152: 34 - 41

[20] Z. Belkhatir and T.M. Laleg-Kirati "High-order sliding mode observer for fractional commensurate linear systems with unknown input" $A u$ tomatica, 2017, 82: $209-217$

[21] R. Martìnez-Guerra, C.D. Cruz-Ancona and C.A. Pérez-Pinacho "Estimators for a class of commensurate fractional order systems with caputo derivative." In: 14th International Conference on Electrical Engineering, Computing Science and Automatic Control (CCE), Mexico City, Mexico, October 2017

[22] S. Saxena, V. Yogesh and P.P. "Arya Reduced-order modeling of commensurate fractional-order system." In: 14th International Conference on Control, Automation, Robotics and Vision (ICARCV), Porto, Portugal, Nov 2016, pp. 1-6 